\section{An Undiagnosed Index Case Leading to a Nosocomial Scabies Outbreak: How Mass Single-Dose Ivermectin Treatment Can Help Control a Nosocomial Epidemic}

To the Editor-Scabies prevalence is increasing in France, particularly in nosocomial settings. ${ }^{1}$ A scabies outbreak was reported in the Oncology and Hematology Department (OHD) of Strasbourg University Hospital following scabies diagnoses in 4 healthcare workers. Initial management was remarkable because no index case was identified. This epidemic lasted 9 weeks from January to April 2015; consequences could have been serious in view of the patients' comorbidities. Here, we describe this outbreak, its course, as well as the containment measures implemented to stop it.

A retrospective and descriptive study of this epidemic was performed. The OHD can accommodate up to 76 inpatients and 47 outpatients in a day hospital. People involved in the epidemic were classified into 2 categories: cases and contacts. Cases were patients or members of the OHD medical team with scabies symptoms who were epidemiologically linked to the index case or to another case. Cases were confirmed by dermatologists or other hospital physicians trained by dermatologists or by a general practitioner. Contacts were either patients cared for by the same medical team as the index case or were members of this medical staff. The epidemic was managed by a crisis team (CT) composed of members of the OHD, hospital administrators, occupational doctors, and hospital hygienists.

Three weeks after the beginning of the epidemic, hospital hygienists were warned that 4 members of the OHD medical staff were suspected of having scabies. From then on, other cases were thoroughly searched for. No index case was identified until 10 days later, when OHD physicians, interrogated by the hospital hygienists, mentioned the case of patient $\mathrm{X}$, a 42-year-old man hospitalized on January 27, 2015, in the OHD for new progression of graft versus host disease $(\mathrm{GvH})$ after regular visits at the day hospital during January. His illness was considered a serious grade IV mucocutaneous $\mathrm{GvH}$ at admission, and he died 9 days after admission. No cutaneous sample was taken to support the scabies diagnosis, but patient $\mathrm{X}$ was the only patient with a clinical profile that could mask crusted scabies. Importantly, his family was found to be chronically infected for 1 year before his admission.

Overall, 35 patients and healthcare professionals were considered secondary cases. Among them, 17 (49\%) were patients, of whom 6 were hospitalized at the same time as patient X; 2 were hospitalized between the time of patient X's death and the outbreak of the epidemic; 6 were outpatients in the 24 hours following his hospitalization in the day hospital. No direct link was found for 3 of the secondary cases. Also, 18 of these 35 cases $(51 \%)$ were healthcare professionals: 11 nurses and 7 nursing aides. No physician was infected. The last scabies case was notified 9 weeks after the beginning of the epidemic.

Once the index case was identified, the contact perimeter was defined on February 27, 2015, as 84 patients hospitalized at the same time as patient $\mathrm{X}, 110$ patients hospitalized after the death of patient $\mathrm{X}$ and at risk of developing scabies by environmental exposure or contact with a secondary case, and 181 members of the OHD medical staff. Because of the appearance of cases among these outpatients, this definition was expanded 2 weeks later to 111 outpatients hospitalized in the 24 hours after patient X's hospitalization in the day hospital. In total, 486 contacts were identified.

To put an end to the epidemic, all cases were treated with 2 doses of oral ivermectin $(200 \mu \mathrm{g} / \mathrm{kg})$. On day 2 of week 4 , the CT decided to treat each contact with 1 dose of oral ivermectin $(200 \mu \mathrm{g} / \mathrm{kg})$ as prophylaxis. Initially, OHD physicians were reluctant to perform this mass treatment, fearing adverse effects in this population, and this led to a delay between notification of cases and mass treatment.

All of the contacts were informed and advised to consult their general practitioner for cutaneous examination and prophylactic treatment. Each contact's rehospitalization status was tagged by the hospital admissions software; their treated or nontreated status was checked at subsequent admission; and ivermectin was administered if necessary. As recommended, ${ }^{2}$ contact precautions were applied to all cases and contacts up to 48 hours after the end of the treatment. These measures stopped the epidemic in 62 days. No adverse effect was reported.

This epidemic emphasizes the difficulties encountered in the management of nosocomial scabies outbreaks. Firstly, multiple and clustered scabies cases, including staff members, should prompt an active search for an index case suffering from crusted scabies, which can be confused with other cutaneous diseases such as $\mathrm{GvH} .^{3}$

Prophylactic treatment for contacts, as recommended by French guidelines, ${ }^{2}$ was delayed due to the reluctance of the medical team to proceed with mass administration of ivermectin because of concerns about side effects. In addition, 15 secondary cases were identified between the hospital hygienist's decision to treat and the actual initiation of treatment nearly 2 weeks later (Figure 1).

The extended contact perimeter emphasizes that contacts from all units must be treated, including outpatients. Although prophylactic treatment was delayed, it was efficient in this context has been observed previously. ${ }^{4}$

A single-dose treatment strategy for contacts according to our experience was chosen, ${ }^{4}$ contrary to French guidelines, which recommend 2 doses of ivermectin. ${ }^{2}$ However, our 


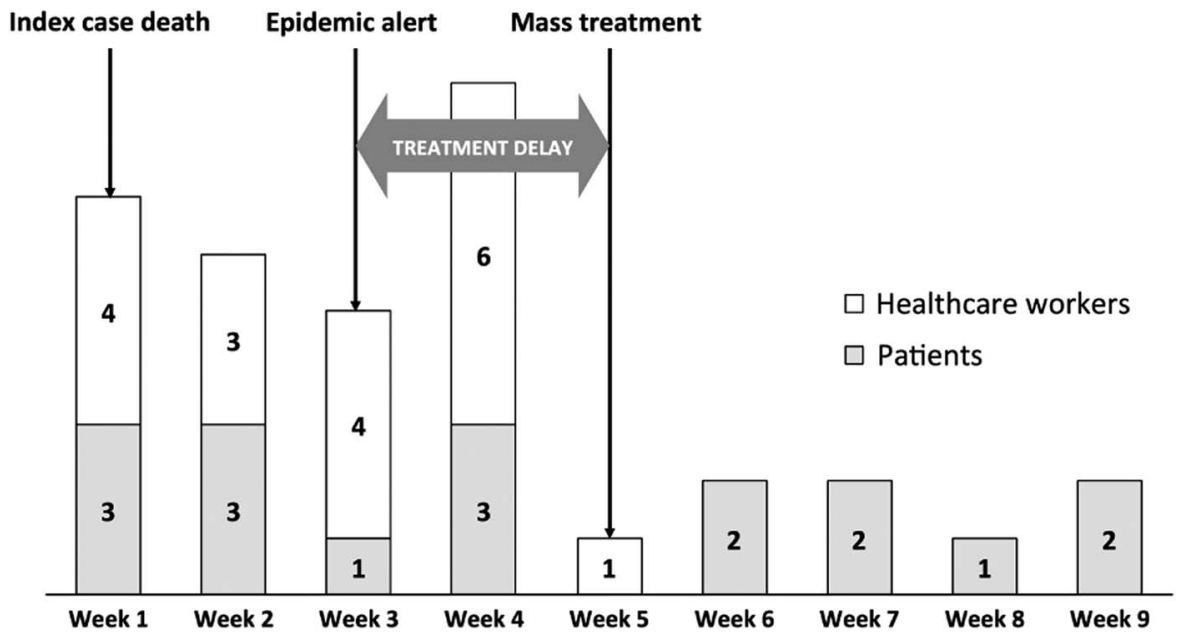

FIGURE 1. Weekly report of the secondary cases among the patient and the medical team during the 9 weeks of the epidemic. Arrows are positioned at 3 key moments. Note the effect of mass treatment on the declaration of new cases.

decision was supported by the result of a recent trial. ${ }^{5}$ The approach taken was also more economic and ensured higher compliance.

This strategy was successful and helped us contain the epidemic reported here in a relatively short period of time compared to previous nosocomial epidemics, which have been known to last up to 1 year. ${ }^{6}$

\section{ACKNOW LEDGMENTS}

We thank Marie-Christine Michellet and Fiona Caulfield for the English correction.

Financial support: No financial support was provided relevant to this article.

Potential conflicts of interest: All authors report no conflicts of interest relevant to this article. implications [in French]. Ann Dermatol Vénéréol 2012;139: 428-434.

2. Commission Specialisée Maladies Transmissibles. Survenue de un ou pluieurs cas de gale. Conduite à tenir. Paris: Haut Conseil de la Santé Publique; 2012.

3. Magee KL, Hebert AA, Rapini RP. Crusted scabies in a patient with chronic graft-versus-host disease. J Am Acad Dermatol 1991;25:889-891.

4. Meyer E-P, Heranney D, Foeglé J, et al. Management of a scabies epidemic in the Strasbourg teaching hospital, France. Médecine Mal Infect 2011;41:92-96.

5. Romani L, Whitfeld MJ, Koroivueta J, et al. Mass drug administration for scabies control in a population with endemic disease. N Engl J Med 2015;373:2305-2313.

6. Vorou R, Remoudaki HD, Maltezou HC. Nosocomial scabies. J Hosp Infect 2007;65:9-14.

\section{Lifesaving Streptococcus bovis Surgical Site Infection}

To the Editor-Surgical site infections (SSIs) are undesired and serious complications following spinal surgery. ${ }^{1,2}$ However, despite full adherence to various protocols for the reduction of SSI, they continue to occur. ${ }^{1,2}$ We report a case of nonpreventable SSI, which certainly complicated the patient's medical management; however, it ultimately saved his life from invasive, metastatic cancer.

This patient was a 54-year-old diabetic male with a 40 pack-year smoking history and chronic low back pain. He underwent decompression and fusion of L4-L5, with pedicle screws, intervertebral body fusion, and graft placement 